\title{
Boundary Representations of $\lambda$-Harmonic and Polyharmonic Functions on Trees
}

\author{
Massimo A. Picardello ${ }^{1} \cdot$ Wolfgang Woess ${ }^{2}$ (D)
}

Received: 14 December 2017 / Accepted: 25 July 2018 / Published online: 11 September 2018

(C) The Author(s) 2018

\begin{abstract}
On a countable tree $T$, allowing vertices with infinite degree, we consider an arbitrary stochastic irreducible nearest neighbour transition operator $P$. We provide a boundary integral representation for general eigenfunctions of $P$ with eigenvalue $\lambda \in C$. This is possible whenever $\lambda$ is in the resolvent set of $P$ as a self-adjoint operator on a suitable $\ell^{2}$-space and the diagonal elements of the resolvent ("Green function") do not vanish at $\lambda$. We show that when $P$ is invariant under a transitive (not necessarily fixed-point-free) group action, the latter condition holds for all $\lambda \neq 0$ in the resolvent set. These results extend and complete previous results by Cartier, by Figà-Talamanca and Steger, and by Woess. For those eigenvalues, we also provide an integral representation of $\lambda$-polyharmonic functions of any order $n$, that is, functions $f: T \rightarrow \mathbb{C}$ for which $(\lambda \cdot I-P)^{n} f=0$. This is a far-reaching extension of work of Cohen et al., who provided such a representation for the simple random walk on a homogeneous tree and eigenvalue $\lambda=1$. Finally, we explain the (much simpler) analogous results for "forward only" transition operators, sometimes also called martingales on trees.
\end{abstract}

Keywords Tree $\cdot$ Stochastic transition operator $\cdot \lambda$-harmonic functions $\cdot$ Polyharmonic functions - Martin kernel - Boundary integral

Mathematics Subject Classification (2010) 31C20 - 05C05 $\cdot 28 \mathrm{~A} 25 \cdot 60 \mathrm{G} 50$

Supported by Austrian Science Fund projects FWF P24028 and W1230. The first author acknowledges support as a distinguished visiting scientist at TU Graz, and partial suppport from MIUR Excellence Department Project funds, awarded by MIUR to the Department of Mathematics, University of Rome Tor Vergata, CUP E83C18000100006.

Wolfgang Woess

woess@tugraz.at

Massimo A. Picardello

picard@axp.mat.uniroma2.it

1 Dipartimento di Matematica, Università di Roma "Tor Vergata", I-00133 Rome, Italy

2 Institut für Diskrete Mathematik, Technische Universität Graz, Steyrergasse 30, A-8010 Graz, Austria 


\section{Introduction}

Let $T$ be a countable tree without leaves (vertices with only one neighbour). We allow vertices with countably many neighbours. On $T$, we consider a random walk which is of nearest neighbour type, that is, the transition probabilities $p(x, y)$ are $>0$ if and only if $x$ and $y$ are neighbours. We consider general eigenfunctions of the transition operator acting on functions $f: T \rightarrow \mathbb{C}$ by

$$
P f(x)=\sum_{y} p(x, y) f(y) .
$$

When this is an infinite summation, $f$ is required to be such that the series converges absolutely. For $\lambda \in \mathbb{C}$, a $\lambda$-harmonic function $h: T \rightarrow \mathbb{C}$ is one that satisfies $P h=\lambda \cdot h$. If we regard $\Delta=P-I$ as a discrete Laplace operator (where $I$ is the identity operator), then $h$ is an eigenfunction of the Laplacian with eigenvalue $\lambda-1$.

First of all, if $\lambda$ is real and $\lambda>\rho(P)$, the spectral radius of the random walk, then every positive $\lambda$-harmonic function has a unique integral representation over the boundary at infinity of $T$ with respect to the $\lambda$-Martin kernel, in analogy with the Poisson representation of positive eigenfunctions of the Laplacian on the unit disk. The same holds for $\lambda=\rho(P)$ in case $P$ is $\rho$-transient.

Furthermore, for those eigenvalues one has a similar integral representation for any real (or complex) eigenfunction, where the integral of the Martin kernel is taken with respect to a distribution, that is, a finitely additive signed measure which is defined on the collection of all boundary arcs. However, this distribution does in general not extend to a $\sigma$-additive measure on the Borel $\sigma$-algebra of the boundary.

When $T$ is locally finite and $\lambda>\rho(P)$ (or $=\rho(P)$ in the $\rho$-transient case), the above integral representation results are comprised in the seminal paper of Cartier [4]; see also Picardello and Woess [13]. The analogous results in the non-locally finite case is comprised in work of Cartwright et al. [3] (for positive functions) and, for general $\lambda$-harmonic functions, in the textbook of Woess $[15, \S 9 . \mathrm{D}]$, which seemingly has remained unobserved by researchers working in this field.

The first goal of this paper is to formulate and prove this general representation theorem in the greatest possible generality, that is, for arbitrary complex-valued $\lambda$-harmonic functions, where $\lambda \in \operatorname{res}(P)=\mathbb{C} \backslash \operatorname{spec}(P)$, the resolvent set of $P$. Here, $P$ is interpreted as a self-adjoint, bounded operator on $\ell^{2}(T, \mathrm{~m})$, where $\mathrm{m}$ is the measure on the vertex set which makes $P$ reversible, that is, $\mathrm{m}(x) p(x, y)=\mathrm{m}(y) p(y, x)$ for all $x, y \in T$. That measure is unique up to normalisation. There is one restriction for the general representation, namely, that $\lambda$ has to be such that the diagonal matrix elements $G(x, x \mid \lambda)$ of the resolvent operator $\mathfrak{G}(\lambda)=(\lambda \cdot I-P)^{-1}$ do not vanish. This holds always when $|\lambda| \geq \rho(P)$. (The use of the letter $\mathfrak{G}$ is motivated by the usual name "Green function" for its matrix elements.) We show that the condition on $\lambda$ allows us to construct the general analogue of the $\lambda$-Martin kernel. Our corresponding integral representation is Theorem 3.2 below. Among other, this generalises a similar result of Figà-Talamanca and Steger [8] concerning the case when $T$ is the regular, locally finite tree which is the Cayley graph of the group $\left\langle a_{1}, \ldots, a_{r} \mid a_{j}=a_{j}^{-1}, j=1, \ldots, r\right\rangle$, and when $P$ is invariant under that group.

Our Theorem 3.2 does not require any group structure. But in addition, we also study in more detail the specific case where $T$ is not necessarily locally finite and $P$ is invariant under the action of an arbitrary group of automorphisms of $T$ which is not required to act with trivial vertex stabilisers, so that $T$ is not necessarily a Cayley graph of that group. Indeed, its closure will be a locally compact group that may be non-discrete and even 
non-unimodular. In this very general group-invariant situation, we provide an extension of another result of [8], namely, that the Green kernel may vanish on the diagonal only for $\lambda=0$, see Theorem 4.2. Thus, we have the integral representation in the group invariant case for all $\lambda$-harmonic functions, where $\lambda \in \operatorname{res}(P)$, with the only possible exception of $\lambda=0$.

Next, we turn our attention to $\lambda$-polyharmonic functions. A function $f: T \rightarrow \mathbb{C}$ is $\lambda$-polyharmonic of order $n$, if

$$
(\lambda \cdot I-P)^{n} f=0 .
$$

If $n=1$, this means that $f$ is $\lambda$-harmonic. If $n=2$ this means that $\lambda \cdot f-P f$ is $\lambda$-harmonic, and so on. (Again, in the non-locally finite case, $f$ is required to be such that the involved sums converge absolutely.)

In the setting of the classical Laplacian $\Delta$ on a Euclidean domain, polyharmonic functions are functions for which $\Delta^{n} h \equiv 0$. Their study goes back to work in the $19^{\text {th }}$ century, see e.g. Almansi [1]. A basic reference is the monograph by Aronszajn et al. [2], and there is ongoing study. The discrete analogue of polyharmonic functions on trees $(\lambda=1)$ was studied in a long paper by Cohen et al. [5]. For the special case of the simple random walk on a locally finite, homogeneous tree, they provide a boundary integral representation for polyharmonic functions. Here, we provide a far-reaching generalisation: in Theorem 5.3 we explain how this can by achieved more directly for arbitrary $\lambda$-polyharmonic functions in the general setting of a nearest neighbour random walk on a countable tree $T$ (locally finite or not), whenever $\lambda \in \operatorname{res}(P)$ fulfils the above condition that $G(x, x \mid \lambda) \neq 0$ for every $x \in T$.

Finally, in the Section 6, we explain how all the above results are obtained very easily for "forward only" transition operators on rooted countable trees, see in particular Proposition 6.2. In this case, the only exceptional eigenvalue is $\lambda=0$.

\section{Basic Facts}

We briefly recall the basic ingredients. First of all, a tree $T$ is a connected graph without circuits. All our trees are supposed to be countable. For two vertices $x, y \in T$, we write $x \sim y$ if they are neighbours. The degree of $x$ is its number of neighbours; it may be infinite. Given any pair of vertices $x, y$, the geodesic or geodesic path from $x$ to $y$ is the unique shortest path $\pi(x, y)$ from $x$ to $y$, and the distance $d(x, y)$ is the length (number of edges) of $\pi(x, y)$.

A ray or geodesic ray in $T$ is a sequence $\left[x_{0}, x_{1}, x_{2}, \ldots\right]$ such that $x_{i-1} \sim x_{i}$ and all $x_{i}$ are distinct. Two rays are equivalent, if they differ by finitely many initial vertices. An end of $T$ is an equivalence class of rays. For any vertex $x$ and end $\xi$, there is a unique ray $\pi(x, \xi)$ which starts at $x$ and represents $\xi$. We write $\partial T$ for the set of all ends of $T$. For $x, y \in T$ with $x \neq y$, the branch or cone $T_{x, y}$ is the subtree spanned by all vertices $w$ with $y \in \pi(x, w)$, and the boundary arc $\partial T_{x, y}$ is the set of all ends which have a representative ray in $T_{x, y}$;

We set $\widehat{T}=T \cup \partial T$ and $\widehat{T}_{x, y}=T_{x, y} \cup \partial T_{x, y}$. We equip $\widehat{T}$ with the discrete topology on the vertex set, while a neighbourhood base of $\xi \in \partial T$ is given by the collection of all $\widehat{T}_{x, y}$ which contain $\xi$. (Here, we may fix $x$ and vary only $y \neq x$.) We obtain a metrisable space which is compact precisely when $T$ is locally finite (all vertex degrees are finite). Otherwise, it is not complete. Following an idea of Soardi, this defect can be overcome by introducing additional boundary points, one associated with each vertex of infinite degree, see Cartwright et al. [3] or the exposition in [15, §9.B]. 
In order to describe convergence to ends, we choose a root vertex $o \in T$. For any pair of elements $v, w \in \widehat{T}$, their confluent $v \wedge w$ with respect to $o$ is the last common vertex on the geodesics $\pi(o, v)$ and $\pi(o, w)$. Then a sequence $\left(x_{n}\right)$ in $T$ converges to an end $\xi$ if and only if $\left|x_{n} \wedge \xi\right| \rightarrow \infty$, where $|x|=d(x, o)$.

Next, let us turn to the random walk. Let $X_{n}$ be the random position at time $n \geq 0$. The $n$-step transition probability $p^{(n)}(x, y)=\operatorname{Pr}\left[X_{n}=y \mid X_{0}=x\right]$ is the $(x, y)$-element of the matrix power $P^{n}$, with $P^{0}=I$, the identity matrix. The spectral radius

$$
\rho=\rho(P)=\limsup _{n \rightarrow \infty} p^{(n)}(x, y)^{1 / n}
$$

is independent of $x$ and $y$. Let $\lambda \in \mathbb{C}$ be such that $|\lambda|>\rho$. The associated Green function is

$$
G(x, y \mid \lambda)=\sum_{n=0}^{\infty} p^{(n)}(x, y) \lambda^{-n-1}
$$

The series converges absolutely. It may or may not converge at $\lambda=\rho$, in which case we say that the random walk is $\rho$-transient, and $\rho$-recurrent, respectively. Next, let us write

$$
f^{(n)}(x, y)=\operatorname{Pr}\left[X_{n}=y, X_{k} \neq y \text { for } k<n \mid X_{0}=x\right]
$$

for the probability that the random walk starting at $x$ reaches $y$ at time $n$, but not before. We set

$$
F(x, y \mid \lambda)=\sum_{n=0}^{\infty} f^{(n)}(x, y) \lambda^{-n} \quad \text { and } \quad U(x, x \mid \lambda)=\sum_{v} p(x, v) F(v, x \mid \lambda) .
$$

If we write $U(x, x \mid \lambda)$ as a power series in $\lambda^{-1}$, the coefficient of $\lambda^{-n}$ is the probability that the first return to the starting point $x$ occurs at time $n(n \geq 1)$. These series certainly converge for $|\lambda|>\rho$, since this is true for the Green function. The following crucial lemma is specific to trees.

Lemma 2.1 The following identities hold for $|\lambda|>\rho$ as well as for $\lambda= \pm \rho$.

(a) For any geodesic path $\left[x_{0}, x_{1}, \ldots, x_{k}\right]$,

$$
F\left(x_{0}, x_{k} \mid \lambda\right)=F\left(x_{0}, x_{1} \mid \lambda\right) F\left(x_{1}, x_{2} \mid \lambda\right) \cdots F\left(x_{k-1}, x_{k} \mid \lambda\right)
$$

(b) For any pair of neighbours $x, y$, we have $F(x, y \mid \lambda) \neq 0$ and

$$
\lambda F(x, y \mid \lambda)=p(x, y)+\sum_{v \neq y} p(x, v) F(v, x \mid \lambda) F(x, y \mid \lambda) .
$$

(c) For any pair of neighbours $x, y$, we have

$$
\sum_{v} p(x, v)|F(v, x \mid \lambda)| \leq|\lambda| \quad \text { and }|F(x, y \mid \lambda) F(y, x \mid \lambda)| \leq 1,
$$

with equality only for $\lambda= \pm \rho$ in the $\rho$-recurrent case, and

$$
G(x, x \mid \lambda)=\frac{1}{\lambda-U(x, x \mid \lambda)}=\frac{F(x, y \mid \lambda) / p(x, y)}{1-F(x, y \mid \lambda) F(y, x \mid \lambda)} .
$$

(d) For arbitrary $x, y \in T$,

$$
G(x, y \mid \lambda)=F(x, y \mid \lambda) G(y, y \mid \lambda) .
$$

In case of vertices $x$ with infinite degree, the sums appearing in (b) and (c) converge absolutely. 
Proof For (a) and the formula displayed in (b), see e.g. [15, Prop. 9.3], while the first of the two displayed formulas for $G(x, x \mid \lambda)$ in (c), as well as (d) are valid for general Markov chains [15, Thm 1.38].

Regarding (c), as mentioned above, the function $U(x, x \mid \lambda)$ is a power series in $\lambda^{-1}$ whose coefficients are non-negative. It converges for $|\lambda|>\rho$, and for positive $\lambda \in[\rho,+\infty)$, it is continuous and decreasing with value 0 at $+\infty$. If one had $U\left(x, x \mid \lambda_{0}\right)=\lambda_{0}$ for some positive $\lambda_{0}>\rho$, then $G(x, x \mid \lambda)$ would have a pole at $\lambda_{0}$, in contradiction with the fact that it is analytic for $|\lambda|>\rho$. Thus $U(x, x \mid \lambda)<\lambda$ for every real $\lambda>\rho$, and $U(x, x \mid \rho)=U(x, x \mid-\rho) \leq \lambda$ with equality precisely when the random walk is $\rho$-recurrent. Consequently, for complex $\lambda$ with $|\lambda|>\rho$, and even for $|\lambda| \geq \rho$ in the $\rho$-transient case, we have

$$
\sum_{v} p(x, v)|F(v, x \mid \lambda)| \leq U(x, x|| \lambda \mid)<|\lambda| .
$$

This proves the first inequality stated in (c), and it shows that for $|\lambda|=\rho$, also $|F(x, y \mid \lambda)| \leq$ $F(x, y \mid \rho)<\infty$.

It also implies that in the context of (b), one has for complex $|\lambda|>\rho$, resp., for $|\lambda| \geq \rho$ in the $\rho$-transient case that

$$
\sum_{v \neq y} p(x, v)|F(v, x \mid \lambda)|<|\lambda|,
$$

which in turn implies $F(x, y \mid \lambda) \neq 0$, as stated in (b).

These arguments also comprise the statement on absolute convergence.

Finally, for the second inequality and the formula for $G(x, x \mid \lambda)$ in (c), we use (b) to see that

$$
F(x, y \mid \lambda)(\lambda-U(x, x \mid \lambda))=p(x, y)(1-F(x, y \mid \lambda) F(y, x \mid \lambda)) .
$$

This yields the displayed formula, as well as the second inequality in the same way as the first one.

At this point, we can define the $\lambda$-Martin kernel by

$$
K(x, w \mid \lambda)=\frac{G(x, x \wedge w \mid \lambda)}{G(o, x \wedge w \mid \lambda)}=\frac{F(x, x \wedge w \mid \lambda)}{F(o, x \wedge w \mid \lambda)}, \quad x \in T, w \in \widehat{T} .
$$

For fixed $x$, it is continuous in the second variable. It is an easy exercise to derive from Lemma 2.1 that for any $\xi \in \partial T$, the function $x \mapsto K(x, \xi \mid \lambda)$ is a $\lambda$-harmonic function (see also below):

$$
\sum_{y \sim x} p(x, y) K(y, \xi \mid \lambda)=\lambda K(x, \xi \mid \lambda) \quad \text { for every } x \in T .
$$

Note that when $x$ has infinite degree, this sum does indeed converge absolutely by Lemma 2.1. The following is well known.

Proposition 2.2 For real $\lambda>\rho$, as well as for $\lambda=\rho$ in the $\rho$-transient case, every positive $\lambda$-harmonic function $h$ has a unique Poisson-Martin integral representation

$$
h(x)=\int_{\partial T} K(x, \xi \mid \lambda) d \nu^{h}(\xi),
$$

where $v^{h}$ is a non-negative Borel measure on $\partial T$ with total mass $h(o)$.

For the case when $T$ is locally finite, this as well as the identities of Lemma 2.1 are comprised in the influential and elegant paper of Cartier [4]. The extension to the 
non-locally finite case goes back to Soardi, see [3] and [15, §9.B+C]. In the last two references, it is assumed that $\lambda=1$. For general positive $\lambda$ and $h$, the result is obtained by applying the standard case to the $h$-process, whose transition probabilities are $p_{h}(x, y)=$ $p(x, y) h(y) /(\lambda h(x))$.

As outlined in the introduction, one of the aims of this paper is to obtain an integral representation for all $\lambda$-harmonic functions for arbitrary $\lambda \in \operatorname{res}(P)=\mathbb{C} \backslash \operatorname{spec}(P)$ up to some possible exceptional values. Proposition 2.2 will follow from that general result. The spectrum $\operatorname{spec}(P) \subset[-\rho, \rho]$ is going to be defined in the next part, which is going to be important for all subsequent considerations.

Remark 2.3 (Analytic continuation) We define a measure $\mathrm{m}$ on $T$ as follows:

$$
\text { for } x \in T \text { with } \pi(o, x)=\left[x_{0}, x_{1}, \ldots, x_{k}\right], \quad \mathrm{m}(x)=\frac{p\left(x_{0}, x_{1}\right) \cdots p\left(x_{k-1}, x_{k}\right)}{p\left(x_{1}, x_{0}\right) \cdots p\left(x_{k}, x_{k-1}\right)} \text {. }
$$

In particular, $\mathrm{m}(o)=1$. We have reversibility: $\mathrm{m}(x) p(x, y)=\mathrm{m}(y) p(y, x)$ for all $x, y$. Thus, $P$ acts as a self-adjoint operator on the Hilbert space $\ell^{2}(T, \mathrm{~m})$ of all functions $f$ : $T \rightarrow \mathbb{R}$ with $\langle f, f\rangle<\infty$, where

$$
\langle f, g\rangle=\sum_{x} f(x) g(x) \mathrm{m}(x) .
$$

It is well-known that the spectral radius $(=$ norm) of this operator is $\rho$, and that $\operatorname{spec}(P)$ is symmetric around the origin. Now, for $\lambda \in \mathbb{C}$ with $|\lambda|>\rho$, the function $G(x, y \mid \lambda)$ is the $(x, y)$-matrix element of the resolvent operator $\mathfrak{G}(\lambda)=(\lambda I-P)^{-1}$ on $\ell^{2}(T, \mathrm{~m})$. Therefore, it extends analytically to the resolvent set $\operatorname{res}(P) \subset \mathbb{C} \backslash[-\rho, \rho]$.

In particular, we note at this point that for $\lambda \in \operatorname{res}(P)$, the function $G(x, x \mid \lambda)$ has no pole, so that by continuity we see from Lemma 2.1 and its proof that

$$
|U(x, x \mid \lambda)|<|\lambda|, G(x, y \mid \lambda) \neq 0 \text { and }|F(x, y \mid \lambda) F(y, x \mid \lambda)|<1
$$

for $x \sim y$ and $|\lambda|>\rho$, and also for $|\lambda|=\rho$ in the $\rho$-transient case. Now,

$$
F(x, y \mid \lambda)=G(x, y \mid \lambda) / G(y, y \mid \lambda) \quad \text { and } \quad U(x, x \mid \lambda)=\lambda-1 / G(x, x \mid \lambda)
$$

extend to meromorphic functions in $\lambda$ on $\operatorname{res}(P)$. We conclude from Lemma 2.1 that for neighbours $x, y \in T$,

$$
\text { if } \begin{gathered}
G(x, x \mid \lambda) G(y, y \mid \lambda) \neq 0 \text { then } U(x, x \mid \lambda) \text { and } F(x, y \mid \lambda) \text { are analytic at } \lambda, \\
F(x, y \mid \lambda) \neq 0 \text { and } F(x, y \mid \lambda) F(y, x \mid \lambda) \neq 1
\end{gathered}
$$

The case when $G\left(x, x \mid \lambda_{0}\right)=0$ is quite special. We know from Lemma 2.1(c) and Eq. 2.6 that $G(x, x \mid \lambda) \neq 0$ for $\lambda \in \mathbb{C}$ with $|\lambda|>\rho$. Replacing $F(x, y \mid \lambda)$ by $G(x, y \mid \lambda) / G(y, y \mid \lambda)$ and $F(y, x \mid \lambda)$ by $G(y, x \mid \lambda) / G(x, x \mid \lambda)$, we can transform the second identity of Lemma 2.1(c) into

$$
\frac{G(x, y \mid \lambda)}{p(x, y)}=G(x, x \mid \lambda) G(y, y \mid \lambda)-G(x, y \mid \lambda) G(y, x \mid \lambda)=\frac{G(y, x \mid \lambda)}{p(y, x)} .
$$

A priori, this holds for $|\lambda|>\rho$, but by analytic continuation, it must also hold in all of res $(P)$. Reordering the terms,

$$
G(x, y \mid \lambda)\left(\frac{1}{p(x, y)}+G(y, x \mid \lambda)\right)=G(x, x \mid \lambda) G(y, y \mid \lambda)
$$

Thus, if $G\left(x, x \mid \lambda_{0}\right)=0$ for some $\lambda_{0} \in \operatorname{res}(P)$, then for any $y \sim x$,

$$
p(x, y) G\left(y, x \mid \lambda_{0}\right)=p(y, x) G\left(x, y \mid \lambda_{0}\right) \in\{0,-1\} .
$$


Now, for any $\lambda \in \operatorname{res}(P)$, we have that $G(\cdot, x \mid \lambda) \in \ell^{2}(T, \mathrm{~m})$ and $P G(\cdot, x \mid \lambda)=$ $\lambda G(\cdot, x \mid \lambda)-\delta_{x}$. In particular, when $G\left(x, x \mid \lambda_{0}\right)=0$ (which can only happen for $|\lambda|<\rho$ )

$$
\sum_{y} p(x, y) G\left(y, x \mid \lambda_{0}\right)=-1,
$$

whence $p(x, y) G\left(y, x \mid \lambda_{0}\right)=p(y, x) G\left(x, y \mid \lambda_{0}\right)$ must have value -1 for precisely one neighbour of $x$, while the value is 0 for all other neighbours. In presence of invariance of the transition probabilities under a transitive action of a group of automorphisms of trees, this fact can be used to exclude that the degenerate case $G\left(x, x \mid \lambda_{0}\right)=0$ can occur, see further below.

\section{The General Poisson-Martin Integral Representation on Trees}

In view of last considerations on analytic continuation, we now define

$$
\operatorname{res}^{*}(P)=\{\lambda \in \operatorname{res}(P): G(x, x \mid \lambda) \neq 0 \text { for all } x\} .
$$

Equation 2.7 tells us that for any $\lambda \in \operatorname{res}^{*}(P)$, we can form the $\lambda$-Martin kernel (2.4), because the denominator does not vanish. By our assumptions, $P K(\cdot, w \mid \lambda)$ is well defined as a function of the first variable. That is, even at vertices with infinite degree, the involved sum is absolutely convergent, and for $\xi \in \partial T$, Eq. 2.5 is valid even when $\lambda \in \operatorname{res}^{*}(P)$ with $|\lambda| \leq \rho$. Indeed, to be precise, write

$$
K(x, \xi \mid \lambda)=\frac{G(x, v \mid \lambda)}{G(o, v \mid \lambda)}, \quad \text { where } v \in \pi(o, \xi), v^{-}=x \wedge \xi
$$

so that we can compute in $\ell^{2}(T, \mathrm{~m})$

$$
P K(x, \xi \mid \lambda)=\frac{1}{G(o, v \mid \lambda)} P \mathfrak{G}(\lambda) \mathbf{1}_{v}(x)=\frac{1}{G(o, v \mid \lambda)} \lambda \mathfrak{G}(\lambda) \mathbf{1}_{v}(x) .
$$

We now consider distributions. For any $x \in T \backslash\{o\}$, is predecessor $x^{-}$is the neighbour of $x$ which is closer to $o$. We write $T_{x}=T_{o, x}$, as well as $T_{o}=T$. A complex distribution on the collection of all boundary arcs

$$
\mathcal{F}_{o}=\left\{\partial T_{x}: x \in T\right\}
$$

is a set function $v: \mathcal{F}_{o} \rightarrow \mathbb{C}$ such that for every $x$,

$$
v\left(\partial T_{x}\right)=\sum_{y: y^{-}=x} v\left(\partial T_{y}\right) .
$$

When $\operatorname{deg}(x)=\infty$, we require that this sum converges absolutely. Note that this does in general not imply that $v$ extends to a $\sigma$-additive distribution on the Borel $\sigma$-algebra of $\partial T$. However, $v$ clearly extends to the collection of all boundary arcs $\partial T_{x, y}$, where $x, y \in T$ $(x \neq y)$. Indeed, if $o \notin T_{x, y}$ then $\partial T_{x, y}=\partial T_{y} \in \mathcal{F}_{o}$. If $o \in T_{x, y}$ then $\partial T \backslash \partial T_{x, y}=$ $\partial T_{y, x}=\partial T_{x}$ and we can define $v\left(\partial T_{x, y}\right)=v(\partial T)-v\left(\partial T_{x}\right)$. In particular, after a change of the root from $o$ to $o^{\prime}$, the distribution satisfies (3.2) also on $\mathcal{F}_{o^{\prime}}$.

A locally constant function on $T$ is a function $f: T \rightarrow \mathbb{C}$ such that the set of edges

$$
E_{f}=\{[x, y] \in E(T): f(x) \neq f(y)\} \text { is finite. }
$$

The union of all geodesic segments which connect $o$ to the endpoints of the edges in $E_{f}$ forms a finite subtree $\tau_{f}$ of $T$. If $\tau$ is any finite subtree of $T$ containing $o$, and $x \in \tau$, then let

$$
S_{\tau}(x)=\left\{y \in \tau: y^{-}=x\right\},
$$


a finite set, possibly empty (the successors of $x$ in $\tau$ ). If $\tau=\tau_{f}$ for $f$ as above, then $f$ constant on each set $T_{x} \backslash \bigcup\left\{T_{y}: y \in S_{\tau}(x)\right\}$. In particular, $f$ extends to a continuous function on $\partial T$. We also call the resulting function on $\partial T$ locally constant. Thus, a function $\varphi$ on $\partial T$ is locally constant if there is a finite subtree $\tau$ of $T$ which contains $o$ such that

$$
\varphi \text { has constant value on each set } \partial T_{x} \backslash \bigcup\left\{\partial T_{y}: y \in S_{\tau}(x)\right\}, x \in \tau .
$$

We shall denote that value by $\varphi_{x}$.

This definition is clearly independent of the choice of the root, and if $\tau^{\prime}$ is another finite subtree which fulfils the same requirement for $\varphi$, then so does the union $\tau \cup \tau^{\prime}$. We can now define

$$
\int_{\partial T} \varphi d v=\sum_{x \in \tau} \varphi_{x}\left(v\left(\partial T_{x}\right)-\sum_{y \in S_{\tau}(x)} v\left(\partial T_{y}\right)\right) .
$$

Equation 3.2 guarantees that this definition does not depend on the specific choice of the finite tree $\tau$ for which (3.3) holds. It also does not depend on the choice of the root $o$. Consequently, the integral is linear on the vector space of all locally constant functions on $\partial T$.

We return to the $\lambda$-Martin kernel, where $\lambda \in \operatorname{res}^{*}(P)$.

Now let $x \in T$ and $\pi(o, x)=\left[o=x_{0}, x_{1}, \ldots, x_{k}=x\right]$. Then $x \wedge \xi \in\left\{x_{0}, x_{1}, \ldots, x_{k}\right\}$ for every $\xi \in \partial T$, and

$$
K(x, \xi \mid \lambda)=K\left(x, x_{i} \mid \lambda\right) \quad \text { when } \quad \begin{cases}\xi \in \partial T_{x_{i}} \backslash \partial T_{x_{i+1}}, & i \leq k-1, \\ \xi \in \partial T_{x_{k}}, & i=k .\end{cases}
$$

Thus, $\varphi=K(x, \cdot \mid \lambda)$ is locally constant on $\partial T$, and we can use $\pi(o, x)$ as a tree $\tau$ for which (3.3) holds. We summarize:

Proposition 3.1 For a distribution $v$ as above, the function

$$
h(x)=\int_{\partial T} K(x, \xi \mid \lambda) d \nu(\xi)
$$

is a $\lambda$-harmonic function, and

$$
\begin{aligned}
h(x) & =\sum_{i=0}^{k-1} K\left(x, x_{i} \mid \lambda\right)\left(v\left(\partial T_{x_{i}}\right)-v\left(\partial T_{x_{i+1}}\right)\right)+K(x, x \mid \lambda) v\left(\partial T_{x}\right) \\
& =K(x, o \mid \lambda) v(\partial T)+\sum_{i=1}^{k}\left(K\left(x, x_{i} \mid \lambda\right)-K\left(x, x_{i-1} \mid \lambda\right)\right) v\left(\partial T_{x_{i}}\right) .
\end{aligned}
$$

We call $h$ the Poisson transform of $v$. The fact that $P h=\lambda \cdot h$ follows from Eq. 3.5, using absolute convergence in Eqs. 3.2 and 3.1.

As outlined in the introduction, for real $\lambda>\rho$, the following theorem goes back to [4] in the locally finite case. The below proof transfers [15, Thm. 9.37] to complex $\lambda$; we present its main part here with additional care regarding absolute convergence in the non-locally finite case.

Theorem 3.2 Let $\lambda \in \operatorname{res}^{*}(P)$, or $\lambda= \pm \rho$ in the $\rho$-transient case. A function $h: T \rightarrow \mathbb{C}$ satisfies $P h=\lambda \cdot h$ if and only if it is of the form

$$
h(x)=\int_{\partial T} K(x, \xi \mid \lambda) d v(\xi),
$$


where $v$ is a complex distribution on $\mathcal{F}_{o}$. The distribution $v$ is determined by $h$, that is, $v=v^{h}$, where

$$
v^{h}(\partial T)=h(o) \quad \text { and } \quad v^{h}\left(\partial T_{x}\right)=F(o, x \mid \lambda) \frac{h(x)-F\left(x, x^{-} \mid \lambda\right) h\left(x^{-}\right)}{1-F\left(x^{-}, x \mid \lambda\right) F\left(x, x^{-} \mid \lambda\right)}, x \neq o .
$$

Proof On the basis of Remark 2.3, the following identities are a consequence of Lemma 2.1(c).

$$
\begin{aligned}
G(x, x \mid \lambda) p(x, y) & =\frac{F(x, y \mid \lambda)}{1-F(x, y \mid \lambda) F(y, x \mid \lambda)} \quad \text { and } \\
\lambda G(x, x \mid \lambda) & =1+\sum_{y: y \sim x} \frac{F(x, y \mid \lambda) F(y, x \mid \lambda)}{1-F(x, y \mid \lambda) F(y, x \mid \lambda)},
\end{aligned}
$$

and when $\operatorname{deg}(x)=\infty$, the last sum converges absolutely. Indeed, the first identity is part of Lemma 2.1(c). The second one then follows from the first one by applying the operator identity

$$
P \mathfrak{G}(\lambda)=\mathfrak{G}(\lambda) P=\lambda \cdot \mathfrak{G}(\lambda)-I .
$$

We now show first that if $h$ is $\lambda$-harmonic, then $v^{h}$ as defined in the theorem is indeed a complex distribution on $\mathcal{F}_{o}$, and $h$ is its Poisson transform. We start with the identity

$$
\lambda G(x, x \mid \lambda) h(x)=\sum_{y} G(x, x \mid \lambda) p(x, y) h(y),
$$

and recall that the sum on the right hand side is assumed to converge absolutely, when $\operatorname{deg}(x)=\infty$. Using (3.6), we rewrite this as

$$
\left(1+\sum_{y: y \sim x} \frac{F(x, y \mid \lambda) F(y, x \mid \lambda)}{1-F(x, y \mid \lambda) F(y, x \mid \lambda)}\right) h(x)=\sum_{y: y \sim x} \frac{F(x, y \mid \lambda)}{1-F(x, y \mid \lambda) F(y, x \mid \lambda)} h(y) .
$$

Using the assumption that the involved sums converge absolutely, we can regroup the terms and get

$$
h(x)=\sum_{y: y \sim x} F(x, y \mid \lambda) \frac{h(y)-F(y, x \mid \lambda) h(x)}{1-F(x, y \mid \lambda) F(y, x \mid \lambda)} .
$$

Convergence is again absolute when $\operatorname{deg}(x)=\infty$.

For $x=o$, the last identity says that $v^{h}(\partial T)=\sum_{y \sim o} v^{h}\left(\partial T_{y}\right)$. Suppose that $x \neq o$. Then by Eq. 3.7,

$$
\begin{aligned}
\sum_{y: y^{-}=x} v^{h}\left(\partial T_{y}\right) & =F(o, x \mid \lambda) \sum_{y: y^{-}=x} F(x, y \mid \lambda) \frac{h(y)-F(y, x \mid \lambda) h(x)}{1-F(x, y \mid \lambda) F(y, x \mid \lambda)} \\
& =F(o, x \mid \lambda)\left(h(x)-F\left(x, x^{-} \mid \lambda\right) \frac{h\left(x^{-}\right)-F\left(x^{-}, x \mid \lambda\right) h(x)}{1-F\left(x, x^{-} \mid \lambda\right) F\left(x^{-}, x \mid \lambda\right)}\right) \\
& =F(o, x \mid \lambda) \frac{h(x)-F\left(x, x^{-} \mid \lambda\right) h\left(x^{-}\right)}{1-F\left(x, x^{-} \mid \lambda\right) F\left(x^{-}, x \mid \lambda\right)}=v^{h}\left(\partial T_{x}\right)
\end{aligned}
$$

So $\nu^{h}$ is indeed a signed distribution on $\mathcal{F}_{o}$. We verify that $\int_{\partial T} K(x, \xi \mid \lambda) d \nu^{h}(\xi)=h(x)$. For $x=o$ this is true. Let $x \neq o$. With the notation of Eq. 3.5, we simplify

$$
\left(K\left(x, x_{i} \mid \lambda\right)-K\left(x, x_{i-1} \mid \lambda\right)\right) v^{h}\left(\partial T_{x_{i}}\right)=F\left(x, x_{i} \mid \lambda\right) h\left(x_{i}\right)-F\left(x, x_{i-1} \mid \lambda\right) h\left(x_{i-1}\right),
$$


whence we obtain

$$
\begin{aligned}
\int_{\partial T} K(x, \xi \mid \lambda) d \nu^{h}(\xi) & =K(x, o \mid \lambda) h(o)+\sum_{i=1}^{k}\left(F\left(x, x_{i} \mid \lambda\right) h\left(x_{j}\right)-F\left(x, x_{i-1} \mid \lambda\right) h\left(x_{i-1}\right)\right) \\
& =F(x, x \mid \lambda) h(x)=h(x),
\end{aligned}
$$

as proposed.

Finally, we need to verify that given $v$ and its Poisson transform $h$, we have $v=v^{h}$. We omit this part of the proof, which is precisely as in [15, Thm. 9.37], with the only generalisation that $P h=\lambda \cdot h$, not necessarliy having $\lambda=1$.

Remark 3.3 (on $\sigma$-additivity) If the distribution $v$ on $\mathcal{F}_{o}$ is non-negative, then it extends uniquely to a $\sigma$-additive measure on the Borel $\sigma$-algebra on $\partial T$. This is a consequence of measure theoretic basics, such as the extension theorems of Caratheodory or Kolmogorov. In the context of boundaries of trees, this fact seems less understood, so we give an outline. Let $\mathcal{A}$ be the product $\sigma$-algebra on $\mathbb{N}^{\mathbb{N}}$. It is generated by all cylinder sets

$$
C\left(a_{1}, \ldots, a_{k}\right)=\left\{\left(m_{n}\right) \in \mathbb{N}^{\mathbb{N}}: m_{i}=a_{i}, i=1, \ldots, k\right\},
$$

where $k \geq 1$ and $a_{1}, \ldots, a_{k} \in \mathbb{N}$. We also allow $k=0$ with the corresponding cylinder set being all of $\mathbb{N}^{\mathbb{N}}$.

Now suppose that for each $k \in \mathbb{N}$, we have a probability distribution $p_{k}$ on $\mathbb{N}^{k}$, such that for all $k$ and all $\left(m_{1}, \ldots, m_{k}\right) \in \mathbb{N}^{k}$,

$$
p_{k}\left(m_{1}, \ldots, m_{k}\right)=\sum_{m_{k+1} \in \mathbb{N}} p_{k+1}\left(m_{1}, \ldots, m_{k}, m_{k+1}\right) .
$$

Then there is a unique probability measure $\operatorname{Pr}$ on $\mathbb{N}^{\mathbb{N}}$, such that for every cylinder set,

$$
\operatorname{Pr}\left(C\left(a_{1}, \ldots, a_{k}\right)\right)=p_{k}\left(a_{1}, \ldots, a_{k}\right) .
$$

A nice exposition, given in the context of Martin boundary theory, is due to Dynkin [7]. There are of course various other sources.

How is this related with trees ? Let $\mathbb{N}^{*}=\bigcup_{k=0}^{\infty} \mathbb{N}^{k}$ be the collection of all words over $\mathbb{N}$. For $k=0$, we intend that $\mathbb{N}^{0}$ consists of the empty word $o$, and $\mathbb{N}^{k}$ is the collection of all words of length $k$. We put a tree structure on $T=\mathbb{N}^{*}$, where the root is the empty word $o$, and neighbourhood is defined in terms of predecessors: for $k \geq 1$, the predecessor of $x=\left(m_{1}, \ldots, m_{k}\right)$ is $x^{-}=\left(m_{1}, \ldots, m_{k-1}\right)$. Then $\partial T=\mathbb{N}^{\mathbb{N}}$ and $\partial T_{x}=C\left(m_{1}, \ldots, m_{k}\right)$. With this identification, a sequence of probability distributions $p_{k}$ on $\mathbb{N}^{k}$ which satisfy (3.8) corresponds bijectively to a measure $v$ on $\mathcal{F}_{o}$ as in Eq. 3.2 by

$$
v\left(\partial T_{x}\right)=p_{k}\left(m_{1}, \ldots, m_{k}\right), \quad \text { where } x=\left(m_{1}, \ldots, m_{k}\right)
$$

If we start with a countable tree $T$ where $\operatorname{deg}(x)=\infty$ for every vertex, then we can choose a root $o$ and label the successors of any vertex by the natural numbers. In this way, we identify $T$ with $\mathbb{N}^{*}$.

A tree which has vertices with finite degree can be embedded into $\mathbb{N}^{*}$ in an obvious way, and then $p_{k}$ will assign value 0 to sequences $\left(m_{1}, \ldots, m_{k}\right)$ which do not correspond to vertices of the tree. This explains that for any countable tree, any non-negative distribution on $\mathcal{F}_{o}$ extends to a Borel measure on the boundary of the tree.

Finally, we may ask when a complex distribution $v$ on $\mathcal{F}_{o}$ which satisfies (3.2) extends to a $\sigma$-additive Borel measure on $\partial T$. In the locally finite case, a necessary and sufficient criterion was given by Cohen et al. [6], which generalises to the non-locally finite case as follows: finite total variation of $v$ is equivalent with existence of some $M<\infty$ such that 
for any sequence of pairwise disjoint boundary arcs $\partial T_{x_{n}}$ (and not only those where all $x_{n}$ have the same predecessor), one has

$$
\sum_{n}\left|v\left(\partial T_{x_{n}}\right)\right| \leq M
$$

(In the locally finite case, it is sufficient that any such series converges, and the upper bound follows.) We omit the details.

Remark 3.4 (Trees with leaves) Suppose that $T$ has leaves, that is, vertices with degree one (besides possibly the root $o$ ). Then the results of this section can be easily adapted to this situation. The leaves have to be incorporated into the boundary $\partial T$. A function $h: T \rightarrow \mathbb{C}$ is $\lambda$-harmonic if $P h(x)=\lambda h(x)$ for every $x \in T^{o}=T \backslash \partial T$. If $v$ is a leaf, then we need to consider $v$ as having no outgoing transition probability, that is, $p(v, v)=1$ and $p\left(v, v^{-}\right)=0$. The representing distribution $v^{h}$ then has mass $v^{h}(v)=F(o, v \mid \lambda) h(v)$ at $v$, while $v^{h}\left(\partial T_{x}\right)$ remains the same when $x \in T^{o}$.

However, as an outlook on Section 5 below, the handling of polyharmonic functions of order $\geq 2$ becomes becomes subtler if there are leaves, as can be seen when studying polyharmonic functions on finite graphs with boundary, see Hirschler and Woess [10]. This is why we restrict ourselves to the situation when there are no leaves.

\section{Invariance under General Transitive Group Actions}

We now study nearest neighbour random walks on countable trees which are invariant under a general subgroup $\Gamma$ of the automorphism group of $T$ which acts transitively on the vertex set. Invariance means that $p(\gamma x, \gamma y)=p(x, y)$ for all $x, y \in T$ and $\gamma \in \Gamma$. Let $I=$ $\Gamma \backslash E(T)$ be the set of orbits of $\Gamma$ on the set of oriented edges of $T$. If $j \in I$ is the orbit (type) of $(x, y) \in E(T)$ then we write $p_{j}=p(x, y)$ and $-j$ for the orbit of $(y, x)$. This is independent of the representative $(x, y)$, and defines an involution: $-(-j)=j$. We have $-j=j$ if and only if there is $\gamma \in \Gamma$ which inverts $(x, y)$. For each $j \in I$ and fixed $x \in T$, we set $d_{j}=|\{y \sim x:(x, y) \in I\}|$. This is independent of $x$ by transitivity of $\Gamma$. When $-j \neq j$ we may well have $d_{-j} \neq d_{j}$. (For example, we may have $I=\{ \pm 1\}$ with $d_{-1}=1$ and $d_{1}=q \in \mathbb{N}$.) The degree of any vertex $x$ is

$$
\operatorname{deg}(x)=\sum_{j \in I} d_{j}, \quad \text { and } \quad \sum_{j \in I} d_{j} p_{j}=1 .
$$

In particular, each $d_{j}$ is finite. Conversely, if we start with a finite or countable set $I$ with an involution $j \mapsto-j$ and a collection $\left(d_{j}\right)_{j \in I}$ of natural numbers, then for the regular tree $T$ with degree $\sum_{j} d_{j} \leq \infty$, there is a group $\Gamma \leq \operatorname{Aut}(T)$ which acts transitively such that $I$ is its set of orbits and the associated cardinalities are $d_{j}$. We postpone the explanation of this and a few other group-theoretic facts to the end of the present section. As a specific example, when $d_{j}=1$ for all $j$ then we can let $\Gamma$ be the discrete group

$$
\left.\Gamma=\left\langle a_{j}, j \in I\right| a_{j}^{-1}=a_{-j} \text { for all } j \in I\right\rangle .
$$

When $j \neq-j$ then we can take just one out of $a_{j}$ and $a_{-j}$ as a free generator; when $j=-j$ then $a_{j}$ is a generator whose square is the group identity. In this example, $\Gamma$ acts transitiviely with trivial stabilisers, and the fact that this provides all possible groups which act in this way on a countable tree is a well-known basic part of Bass-Serre Theory, see Serre [14]. 
Here, we shall always assume that the vertex degree is $\geq 3$, so that our random walk has to be $\rho$-transient by a result of Guivarc'h [9]. The Green function $G(x, x \mid \lambda)=G(\lambda)$ is again independent of $x$, and if $(x, y)$ is an oriented edge of type $j$, then $G(x, y \mid \lambda)=G_{j}(\lambda)$ depends only on $j$. If $G\left(\lambda_{0}\right)=0$ for $\lambda_{0} \in \operatorname{res}(P)$ then there must be a unique $j_{0} \in I$ such that

$$
-j_{0}=j_{0}, \quad d_{j_{0}}=1, \quad p_{j_{0}} G_{j_{0}}\left(\lambda_{0}\right)=-1, \quad \text { and } \quad p_{j} G_{-j}\left(\lambda_{0}\right)=0 \quad \text { for all } j \neq j_{0} .
$$

This follows from the last lines of Remark 2.3. Namely, if we fix $x \in T$ then there is precisely one $y \sim x$ such that $p(x, y) G\left(y, x \mid \lambda_{0}\right)=p(y, x) G\left(x, y \mid \lambda_{0}\right)=-1$, while $p(x, v) G\left(v, x \mid \lambda_{0}\right)=p(v, x) G\left(x, v \mid \lambda_{0}\right)=0$ for all other $v \sim x$.

Thus, if $d_{j} \geq 2$ for all $j$ with $-j=j$, then $G(\lambda) \neq 0$ for all $\lambda \in \operatorname{res}(P)$.

Corollary 4.1 For any nearest neighbour random walk on a finitely or countably generated free group, $G(\lambda) \neq 0$ for all $\lambda \in \operatorname{res}(P)$, so that $\operatorname{res}^{*}(P)=\operatorname{res}(P)$.

By reversibility, we have

$$
p_{j} G_{-j}(\lambda)=p_{-j} G_{j}(\lambda)
$$

and Eq. 2.9 becomes

$$
p_{-j} G_{j}(\lambda)^{2}+G_{j}(\lambda)-p_{j} G(\lambda)^{2}=0 .
$$

When $\lambda>\rho$ is real, the right one among the two solutions of this equation is

$$
G_{j}(\lambda)=\frac{1}{2 p_{-j}}\left(\sqrt{1+4 p_{j} p_{-j} G(\lambda)^{2}}-1\right),
$$

because the functions $G(\lambda)$ and $G_{j}(\lambda)$ are decreasing in those $\lambda$. In other regions of the plane, there may be the minus sign in front of the root. We now show that the only possible $\lambda_{0} \in \operatorname{res}(P)$ for which $G\left(\lambda_{0}\right)=0$ is $\lambda_{0}=0$.

Theorem 4.2 If $0 \neq \lambda \in \operatorname{res}(P)$ then $G(\lambda) \neq 0$, so that $\operatorname{res}(P) \backslash \operatorname{res}^{*}(P) \subset\{0\}$.

Proof We modify and extend an argument of [8, p. 17]. Suppose that $G\left(\lambda_{0}\right)=0$ for some $\lambda_{0} \in \operatorname{res}(P)$. By analyticity, there is an open ball $B$ centred at $\lambda_{0}$ with closure $B^{-} \subset \operatorname{res}(P)$ such that $G(\lambda) \neq 0$ for $\lambda \in B^{-} \backslash\left\{\lambda_{0}\right\}$. By continuity, we may also choose $B$ such that $2|G(\lambda)|<1$ for all $\lambda \in B^{-}$.

We know from Remark 2.3 that Eq. (2.7) holds for those $\lambda$, and as we have observed above, there is precisely one $j_{0} \in I$ such that $p_{-j_{0}} G_{j_{0}}\left(\lambda_{0}\right)=-1$, and by Eq. 4.2 we must have $-j_{0}=j_{0}$ and $d_{j_{0}}=1$.

For all $j \in I_{0}=I \backslash\left\{j_{0}\right\}$, we have $G_{j}\left(\lambda_{0}\right)=0$, so that the right solution of Eq. 4.3 at $\lambda_{0}$ is the one given by Eq. 4.4. By continuity, this must also hold in a neighbourhood of $\lambda_{0}$. On the other hand, by the choice of $B$, the right hand side of Eq. 4.3 is analytic in $B$, so that by analytic continuation, Eq. 4.3 must hold for $G_{j}(\lambda)$ in all of $B$. Analogously,

$$
G_{j_{0}}(\lambda)=\frac{1}{2 p_{-j}}\left(-\sqrt{1+4 p_{j} p_{-j} G(\lambda)^{2}}-1\right) \text { for } \lambda \in B .
$$


We now note that for complex $t$ with $|t|<1$, one has $|\sqrt{1+t}-1|<|t|$. Thus, for $\lambda \in B$ and $j \in I_{0}$, Eq. 4.4 gives $\left|G_{j}(\lambda)\right| \leq 2 p_{j}|G(\lambda)|^{2}$. When in addition $\lambda \neq \lambda_{0}$, setting $F_{j}(\lambda)=G_{j}(\lambda) / G(\lambda)$, we can transform the identity of Lemma 2.1(b) into

$$
\lambda=\frac{G(\lambda)}{G_{j_{0}}(\lambda)}+\sum_{j \in I_{0}} d_{-j} p_{-j} F_{j}(\lambda) .
$$

By the above, for $\lambda \in B$,

$$
\sum_{j \in I_{0}} d_{-j} p_{-j}\left|F_{j}(\lambda)\right| \leq 2|G(\lambda)| \sum_{j \in I_{0}} d_{-j} p_{-j} p_{j}<2|G(\lambda)|,
$$

since $d_{-j} p_{-j} \leq 1$. Thus, when $\lambda \rightarrow \lambda_{0}$, the sum tends to 0 , and we also know that $G(\lambda) / G_{j_{0}}(\lambda) \rightarrow 0$. Therefore $\lambda_{0}=0$, which we have excluded.

Corollary 4.3 In case of group-invariance under a transitive automorphism group of $T$, the integral representation of Theorem 3.2 holds for all eigenvalues $\lambda \in \operatorname{res}(P)$ with the only possible exception of $\lambda=0$.

We remark that it may well happen that 0 is part of the resolvent set of $P$ for certain choices of the probabilities $p_{j}$, see [8].

Remark 4.4 (General transitive group actions) We now add some details on group actions. For any tree (or graph), the automorphism group $\operatorname{Aut}(T)$ of all neighbourhood preserving bijections of the vertex set is equipped with the topology of point-wise convergence. If $T$ is locally finite, then the vertex stabilisers $\{\gamma \in \operatorname{Aut}(T): \gamma x=x\}$ for $x \in T$ are opencompact, so that $\operatorname{Aut}(T)$ is a locally compact, totally disconnected group. When $T$ is not locally finite, this is more delicate. However, given the transition operator, resp. matrix $P=(p(x, y))_{x, y \in T}$, we are interested in the group

$$
\operatorname{Aut}(T, P)=\{\gamma \in \operatorname{Aut}(T): p(\gamma x, \gamma y)=p(x, y) \text { for all } x, y\} .
$$

When $\operatorname{Aut}(T, P)$ acts transitively (even when $T$ is not locally finite), it is closed in the topology of point-wise convergence and acts with open-compact vertex stabilisers whose orbits are all finite, see Kaimanovich and Woess [11, Prop. 2.7]. Hence, if $\Gamma$ is a group as assumed at the beginning of this section, we can pass to its closure in $\operatorname{Aut}(T, P)$, or rather assume right away without loss of generality that $\Gamma=\operatorname{Aut}(T, P)$.

We now address the converse question: we start with a finite or countable set $I$ with an involution $j \mapsto-j$ and a collection $\left(d_{j}\right)_{j \in I}$ of natural numbers and look for a group $\Gamma \leq \operatorname{Aut}(T)$ which acts transitively and realises $I$ as the set of orbits and the associated cardinalities $d_{j}$.

To begin, we fix $j$ together with $d_{j}$ and $d_{-j}$.

Let us first consider the case when, under the given involution, one has $-j \neq j$. Then we consider the regular tree $T_{j}=T\left(d_{j}, d_{-j}\right)$ with degree $d_{j}+d_{-j}$. We orient its edges such that each vertex has $d_{-j}$ ingoing and $d_{j}$ outgoing edges. All edges are given the "colour" $j$. Then we consider the group $\Gamma_{j}$ of all automorphisms of $T_{j}$ which preserve the orientation. This is a closed subgroup of Aut $\left(T_{j}\right)$ which acts transitively, and the stabiliser of any vertex $x$ has two sub-orbits on the neighbours of $x$, namely the forward and the backward neighbours.

Next, consider the special case when $j=-j$ and thus $d_{j}=d_{-j}$. Then we take $T_{j}$ to be the regular tree with degree $d_{j}$ and $\Gamma_{j}=\operatorname{Aut}\left(T_{j}\right)$, its full automorphsim group. (Note that when $d_{j}=d_{-j}=1$, the tree $T_{j}$ consists of two vertices connected by one edge.) The stabiliser of any vertex acts transitively on its neighbours, which is what we need. 
Now, we consider the reduced index set $I^{\prime}$, where for each $j \in I$ with $-j \neq j$, we keep only one of $j$ and $-j$ and eliminate the other one. At this point, we consider the free product graph

$$
T=\underset{j \in I^{\prime}}{*} T_{j}
$$

For this purpose, choose a root $o_{j}$ in each tree $T_{j}$ and set $T_{j}^{\prime}=T_{j} \backslash\left\{o_{j}\right\}$. The vertex set of $T$ then consists of all alternating words over the set $\bigcup_{j \in I^{\prime}} T_{j}^{\prime}$, that is all finite sequences

$$
\begin{aligned}
& x_{1} x_{2} \cdots x_{n}, \quad \text { where } n \geq 0 \\
& \quad \text { and } x_{k} \in T_{j(k)}^{\prime} \text { for some } j(k) \in I^{\prime} \text { with } j(k+1) \neq j(k) .
\end{aligned}
$$

For $n=0$, this is the empty word $o$. The edges of $T$ are as follows for all $n \geq 1$.

(a) A vertex $x_{1} \cdots x_{n-1} x_{n}$ as above is connected by an edge with colour $j(n)$ to $x_{1} \cdots x_{n-1} y_{n}$ if and only if there is an edge from $x_{n}$ to $y_{n}$ in $T_{j(n)}$.

(b) A vertex $x_{1} \cdots x_{n-1}$ is connected by an edge with colour $j(n)$ to $x_{1} \cdots x_{n-1} x_{n}$ if and only if there is an edge from $o_{j(n)}$ to $x_{n}$ in $T_{j(n)}$.

In both cases, the resulting edges inherit their eventual orientation from $T_{j}$.

Thus, $T$ is a tree which contains countably many copies of each $T_{j}$ : any element $x_{1} \cdots x_{n-1}$ with $j(n-1) \neq j$ together with the collection of all $x_{1} \cdots x_{n-1} x_{n}$ with $x_{n} \in T_{j}^{\prime}$ spans such a copy, in which the root of $T_{j}$ corresponds to $x_{1} \cdots x_{n-1}$.

Each $\Gamma_{j}$ embeds into Aut $(T)$ as follows. Let $\gamma \in \Gamma_{j}$ and $x_{1} \cdots x_{n} \in T$ as in Eq. 4.5.

- If $j(1) \neq j$ and $\gamma o_{j}=o_{j}$ then $\gamma\left(x_{1} \cdots x_{n}\right)=x_{1} \cdots x_{n}$, in particular $\gamma o=o$ in $T$.

- If $j(1) \neq j$ and $\gamma o_{j} \neq o_{j}$ then $\gamma\left(x_{1} \cdots x_{n}\right)=\left(\gamma o_{j}\right) x_{1} \cdots x_{n}$.

- If $j(1)=j$ and $\gamma x_{1}=o_{j}$ then $\gamma\left(x_{1} \cdots x_{n}\right)=x_{2} \cdots x_{n}$.

- If $j(1)=j$ and $\gamma x_{1} \neq o_{j}$ then $\gamma\left(x_{1} \cdots x_{n}\right)=\left(\gamma x_{1}\right) x_{2} \cdots x_{n}$.

The subgroup of $\operatorname{Aut}(T)$ generated by $\bigcup_{j \in I^{\prime}} \Gamma_{j}$ preserves the colours and the eventual orientations of the edges. It acts transitively and has all the required properties.

\section{The Integral Representation of $\lambda$-Polyharmonic Functions}

We return to the general setting of Section 2. A $\lambda$-polyharmonic function of order $n \geq 1$ is a function $f: T \rightarrow \mathbb{C}$ such that

$$
(\lambda I-P)^{n} f \equiv 0 .
$$

If $n=1$, this means that $f$ is $\lambda$-harmonic. If $n=2$ this means that $\lambda \cdot f-P f$ is $\lambda$-harmonic, and so on.

In the setting of the classical Laplacian $\Delta$ on a Euclidean domain, polyharmonic functions are functions for which $\Delta^{n} h \equiv 0$. Their study goes back to work in the $19^{\text {th }}$ century, see e.g. Almansi [1]. A basic reference is the monograph by Aronszajn et al. [2], and there is ongoing study.

The discrete analogue of polyharmonic functions on trees $(\lambda=1)$ was studied in a long paper by Cohen et al. [5]. For the case of the simple random walk on a homogeneous tree, they provide a boundary integral representation for polyharmonic functions. Here, we shall explain how this can by achieved more directly for arbitrary $\lambda$-polyharmonic functions in the general setting of a nearest neighbour random walk on a countable tree $T$ (locally finite or not), whenever $\lambda \in$ res* $^{*}(P)$. We know from Lemma 2.1 and Remark 2.3 that all $\lambda \in \mathbb{C} \backslash\{ \pm \rho\}$ with $|\lambda| \geq \rho$ belong to res* $(P)$, and we also know from Theorem 
4.2that in the group-invariant case $\operatorname{res}^{*}(P)$ differs from the resolvent set res $(P)$ at most by $\{0\}$.

The key to our integral representation is the following proposition, where for $x \in T$ and $\xi \in \partial T$, we denote by $K^{(r)}(x, \xi \mid \lambda)$ the $r^{\text {th }}$ derivative of the function $\lambda \mapsto K(x, \xi \mid \lambda)$. Differentiability of that function follows from Eq. 2.4, i.e., the fact that it is the quotient of the Green functions at vertices of $T$ - a property which is specific to the nearest neighbour case.

Proposition 5.1 For $\lambda \in \operatorname{res}^{*}(P)$,

$$
(\lambda I-P) K^{(r)}(\cdot, \xi \mid \lambda)=-r K^{(r-1)}(\cdot, \xi \mid \lambda) .
$$

Therefore, setting

$$
h(x \mid \lambda)=\int_{\partial T} K(\cdot, \xi \mid \lambda) d v(\xi)
$$

its $r^{\text {th }}$ derivative $h^{(r)}(x \mid \lambda)$ with respect to $\lambda$ satisfies

$$
(\lambda I-P) h^{(r)}(\cdot \mid \lambda)=-r h^{(r-1)}(\cdot \mid \lambda) .
$$

Proof Given $x$ and $\xi$, let $c=x \wedge \xi$. We can write $K(x, \xi \mid \lambda)=G(x, c \mid \lambda) / G(o, c \mid \lambda)$, which is legitimate since our $\lambda$ is such that the denominator does not vanish. When $T$ is locally finite, it is obvious that in the identity

$$
P K(x, \xi \mid \lambda)=\lambda K(x, \xi \mid \lambda),
$$

we may exchange the derivatives with respect to $\lambda$ with the application of $P$, even when $T$ is not locally finite. The fact that this is also true in the non-locally finite case follows from Lemma 5.2 below, together with Eq. 3.1.

Next, formula (3.5) of Proposition 3.1 shows that $\int_{\partial T} K(x, \xi \mid \lambda) d \nu(\xi)$ is in fact a finite linear combination of functions $K\left(x, x_{i} \mid \lambda\right)$, so that the second statement is immediate.

Let us now clarify why the derivation and summation in Eq. 3.1 can be exchanged even in the non-locally finite case. We recall (without proof) some basic facts which are part of the functional calculus for the resolvent of a bounded self-adjoint operator on a Hilbert space within our context; see e.g. Kato [12], Yosida [16, Ch. VIII], or various other textbooks.

Lemma 5.2 For $\lambda \in \operatorname{res}(P)$, we have the following for the resolvent operator $\mathfrak{G}(\lambda)=$ $(\lambda I-P)^{-1}$ on $\ell^{2}(T, \mathrm{~m})$.

The operator-valued mapping $\lambda \mapsto \mathfrak{G}(\lambda)$ is analytic, and its $r^{\text {th }}$ derivative $\mathfrak{G}^{(r)}(\lambda)$ with respect to $\lambda$ satisfies

$$
\begin{aligned}
\mathfrak{G}^{(r)}(\lambda) & \left.=(-1)^{r} r !(\mathfrak{G}(\lambda))^{r+1} \text { (operator power of order } r+1\right), \text { and } \\
P \mathfrak{G}^{(r)}(\lambda) & =\frac{d^{r}}{d \lambda^{r}}(P \mathfrak{G}(\lambda))=r \cdot \mathfrak{G}^{(r-1)}(\lambda)+\lambda \cdot \mathfrak{G}^{(r)}(\lambda), \quad r \geq 1 .
\end{aligned}
$$

For $r=1$, the first formula may be seen as a consequence of the resolvent equation

$$
\mathfrak{G}\left(\lambda_{1}\right)-\mathfrak{G}\left(\lambda_{2}\right)=\left(\lambda_{2}-\lambda_{1}\right) \cdot \mathfrak{G}\left(\lambda_{1}\right) \mathfrak{G}\left(\lambda_{2}\right)
$$


Subsequently, one can proceed by induction on $r$. Rewriting the previous equations explicitly in terms of matrix elements of the operators, we have for example

$$
\begin{aligned}
G^{\prime}(x, y \mid \lambda) & =-\sum_{v} G(x, v \mid \lambda) G(v, y \mid \lambda) \quad \text { and } \\
G^{\prime \prime}(x, y \mid \lambda) & =2 \sum_{v, w} G(x, v \mid \lambda) G(v, w \mid \lambda) G(w, y \mid \lambda)
\end{aligned}
$$

as well as

$$
\sum_{v} p(x, v) G^{\prime}(v, y \mid \lambda)=G(x, y \mid \lambda)+\lambda G^{\prime}(x, y \mid \lambda),
$$

etc. This makes clear that Proposition 5.1 is valid in general.

If $h$ is a $\lambda$-harmonic function then by Theorem 3.2 there is a unique distribution $v$ on $\partial T$ such that

$$
h(x)=h(x \mid \lambda)=\int_{\partial T_{x}} K(x, \xi \mid \lambda) d \nu(\xi) .
$$

and we can consider the functions

$$
h^{(r)}(x)=h^{(r)}(x \mid \lambda)=\int_{\partial T_{x}} K^{(r)}(x, \xi \mid \lambda) d \nu(\xi) .
$$

From Proposition 5.1 we infer that

$$
(\lambda I-P)^{r} h^{(r)}=(-1)^{r} r ! \cdot h .
$$

So, since $h$ is $\lambda$-harmonic, the derived function $h^{(r)}$ is $\lambda$-polyharmonic of order $r+1$. This leads us to the main theorem of this section.

Theorem 5.3 For $\lambda \in$ res* $^{*}(P)$, every $\lambda$-polyharmonic function $f$ of order $n$ has an integral representation

$$
f(x)=\sum_{r=0}^{n-1} \int_{\partial T} K^{(r)}(x, \xi \mid \lambda) d v_{r}(\xi),
$$

where the collection of distributions $\left(v_{0}, \ldots, v_{n-1}\right)$ in the sense of Eq. 3.2 is uniquely determined by $f$. Conversely, every function which has an integral representation as above is $\lambda$-polyharmonic of order $n$.

Proof We use induction on $n$. The statement is true for $n=1$ by Theorem 3.2. Now suppose it is true for $n-1$. Let $f$ be polyharmonic of order $n$. Then we have the integral representation of the $\lambda$-harmonic function

$$
h(x)=\frac{(-1)^{n-1}}{(n-1) !}(\lambda I-P)^{n-1} f=\int_{\partial T} K(x, \xi \mid \lambda) d v_{n-1}(\xi)
$$

for the uniquely determined distribution $v_{n-1}$ on $\partial T$. Deriving $n-1$ times with respect to $\lambda$, the indentity (5.1) yields

$$
(\lambda I-P)^{n-1} h^{(n-1)}=(-1)^{n-1}(n-1) ! \cdot h=(\lambda I-P)^{n-1} f .
$$

Thus, the function $f-h^{(n-1)}$ is $\lambda$-polyharmonic of order $n-1$. By the induction hypothesis, it has a unique integral representation

$$
f(x)-h^{(n-1)}(x)=\sum_{r=0}^{n-2} \int_{\partial T} K^{(r)}(x, \xi \mid \lambda) d \nu_{r}(\xi) .
$$


Since

$$
h^{(n-1)}(x)=\int_{\partial T} K^{(n-1)}(x, \xi \mid \lambda) d v_{n-1}(\xi),
$$

the result follows.

The Isotropic Case We now consider the specific case when $T=\mathbb{T}_{q}$ is the homogeneous tree with degree $q+1$, and $P$ is the simple random walk, i.e., $p(x, y)=1 /(q+1)$ when $x \sim$ $y$. In this situation, [5, Thm. 4.1] gives an integral representation of polyharmonic functions for $\lambda=1$. By making use of Theorem 5.3 above, we give a simpler proof of that result, and we also extend it to general eigenvalues $\lambda$. First of all, it is well known at least since [4] (and has been computed again and again by many authors) that $\operatorname{spec}(P)=[-\rho, \rho]$, where $\rho=2 \sqrt{q} /(q+1)$. Thus, by Theorem 4.2, $\operatorname{res}^{*}(P)=\operatorname{res}(P)=\mathbb{C} \backslash[-\rho, \rho]$. For $\lambda \in \operatorname{res}(P)$ and neighbours $x, y \in T$, the function $F(\lambda)=F(x, y \mid \lambda)$ has also been re-computed many times, usually in the variable $z=1 / \lambda$, on the basis of the quadratic equation which results from Lemma 2.1(b). The expression of the $\lambda$-Martin kernel becomes

$$
K(x, \xi \mid \lambda)=F(\lambda)^{\mathfrak{h}(x, \xi)}, \quad \text { where } \quad F(\lambda)=F_{-}(\lambda)=\frac{(q+1) \lambda}{2 q}\left(1-\sqrt{1-\rho^{2} / \lambda^{2}}\right),
$$

and $\mathfrak{h}(x, \xi)=d(x, x \wedge \xi)-d(o, x \wedge \xi)$ is the horocycle index of $x$ with respect to $\xi$. We compute

$$
K^{\prime}(x, \xi \mid \lambda)=K(x, \xi \mid \lambda) \mathfrak{h}(x, \xi) f(\lambda), \quad \text { where } \quad f(\lambda)=-\frac{1}{\lambda \sqrt{1-\rho^{2} / \lambda^{2}}}
$$

From here, we get recursively

$$
\begin{aligned}
& K^{(r)}(x, \xi \mid \lambda)=K(x, \xi \mid \lambda) \sum_{k=1}^{r} \mathfrak{h}(x, \xi)^{k} f_{k, r}(\lambda), \quad \text { where } \\
& f_{1, r}(\lambda)=f^{(r-1)}(\lambda) \quad[\text { derivative of order } r-1], \quad f_{r, r}(\lambda)=f(\lambda)^{r}, \quad \text { and } \\
& f_{k, r}(\lambda)=f_{k, r-1}^{\prime}(\lambda)+f(\lambda) f_{k-1, r-1}(\lambda) \quad \text { for } r \geq 2, k=2, \ldots, r-1 .
\end{aligned}
$$

Now suppose that $f$ is a $\lambda$-polyharmonic function of order $n$ for our simple random walk on the homogeneous tree. Let

$$
f(x)=\int_{\partial T} K(x, \xi \mid \lambda) d v_{0}(\xi)+\sum_{r=1}^{n-1} \int_{\partial T} K^{(r)}(x, \xi \mid \lambda) d v_{r}(\xi)
$$

be its unique integral representation according to Theorem 5.3. By Eq. 5.2, we can rewrite the last sum in terms of new distributions $\bar{v}_{k}$ as

$$
\sum_{k=1}^{n-1} \int_{\partial T} K(x, \xi \mid \lambda) \mathfrak{h}(x, \xi)^{k} d \bar{v}_{k}, \quad \text { where } \quad \bar{v}_{k}=\sum_{r=k}^{n-1} f_{k, r}(\lambda) v_{r} .
$$

Since $f(\lambda) \neq 0$, the upper triangular $(n-1) \times(n-1)$-matrix $A_{n-1}(\lambda)=$ $\left(f_{k, r}(\lambda)\right)_{1 \leq k \leq r \leq n-1}$ is invertible. Thus, we can restate Theorem 5.3 in this case as follows.

Corollary 5.4 For the simple random walk on $T=\mathbb{T}_{q}$ and $\lambda \in \mathbb{C} \backslash[-\rho, \rho]$, let $f$ be a $\lambda$-polyharmonic function of order $n$. Then $f$ has an integral representation

$$
f(x)=\sum_{k=0}^{n-1} \int_{\partial T} K(x, \xi \mid \lambda) \mathfrak{h}(x, \xi)^{k} d \bar{\nu}_{k}(\xi),
$$


where the collection of distributions $\left(\bar{v}_{0}, \ldots, \bar{v}_{n-1}\right)$ in the sense of Eq. 3.2 is uniquely determined by $f$. Conversely, every function of this form is $\lambda$-polyharmonic of order $n$.

(Note that $\bar{v}_{0}=v_{0}$. .) Uniqueness follows from invertibility of $A_{n-1}(\lambda)$. In the special case $\lambda=1$, this corollary is [5, Thm. 4.1]. Indeed, note that for the simple random walk on $\mathbb{T}_{q}$, the $k_{\omega}(v)$ of [5] is $-\mathfrak{h}(v, \omega)$ in our notation, where $v \in T$ and $\omega \in \partial T$. Also, $F(1)=1 / q$, so that Corollary 5.4 yields the representation of [5, Thm. 4.1].

Next, in forthcoming work we shall study the interplay of the boundary integral representation with the limiting behaviour of polyharmonic functions in our general setting.

\section{Remarks on "Forward Only" Transition Operators}

So far we have assumed $p(x, y)>0$ whenever $x \sim y$. On a countable tree $T$ with root vertex $o$ as before, we now consider a different, simpler type of random walks. To keep notation different, we denote the stochastic transition matrix by $Q=(q(x, y))_{x, y \in T}$, assuming that

$$
q(x, y)>0 \Longleftrightarrow x=y^{-} .
$$

The associated random walk $\left(Y_{n}\right)_{n \geq 0}$ starting at $o$ is such that $d\left(Y_{n}, o\right)=n$. The general $n$-step-transition probabilities are

$$
\begin{aligned}
& q^{(n)}(x, y)=q\left(x_{0}, x_{1}\right) q\left(x_{1}, x_{2}\right) \cdots q\left(x_{n-1}, x_{n}\right), \\
& \text { if } y \in T_{x} \text { and } \pi(x, y)=\left[x=x_{0}, x_{1}, \ldots, x_{n}=y\right],
\end{aligned}
$$

while $q^{(n)}(x, y)=0$ in all other cases. Of course, we define $q^{(0)}(x, y)=\delta_{x}(y)$. It is clear that $\left(Y_{n}\right)$ converges almost surely to a $\partial T$-valued random variable $Y_{\infty}$, whose distribution is the Borel measure on $\partial T$ given by

$$
\boldsymbol{v}\left(\partial T_{x}\right)=q^{(n)}(o, x), \quad \text { where } n=|x| .
$$

(Recall that $|x|=d(x, o)$.) In fact, this is precisely the situation defined in Remark 3.3, and we can recover the transition probabilities from $\boldsymbol{v}$ by

$$
q(x, y)=\boldsymbol{v}\left(\partial T_{y}\right) / \boldsymbol{v}\left(\partial T_{x}\right), \quad \text { when } x=y^{-} .
$$

For $\lambda \in \mathbb{C}$, a $\lambda$-harmonic function $h$ is defined as before: $Q h=\lambda \cdot h$, or equivalently,

$$
\sum_{y: y^{-}=x} \boldsymbol{v}\left(\partial T_{y}\right) h(y)=\boldsymbol{v}\left(\partial T_{x}\right) h(x) \text { for every } x \in T .
$$

For $\lambda=1$, such functions are often called martingales, which is completely justified. Indeed, keeping in mind Remark 3.3, we can view the random variables $Y_{n}$ to be defined on the probability space $(\partial T, \boldsymbol{v})$ by $Y_{n}(\xi)=y_{n}$, where $y_{n}$ is the $n^{\text {th }}$ vertex on the ray $\pi(o, \xi)$. The filtration $\left(\mathcal{F}_{n}\right)_{n \in \mathbb{N}}$ of the Borel $\sigma$-algebra of $\partial T$ induced by the stochastic process $\left(Y_{n}\right)$ is the one where $\mathcal{F}_{n}$ is the sub- $\sigma$-algebra generated by the collection of atoms $\left\{\partial T_{x}:|x|=n\right\}$. Then the martingales with respect to this filtration are precisely the sequences $\left(h\left(Y_{n}\right)\right)$, where $Q h=h$; compare with [7] or with [15, §7.C.I]. Analogously, $\lambda$-harmonic functions correspond to $\lambda$-martingales, where

$$
\mathrm{E}_{v}\left[h\left(Y_{n+1}\right) \mid \mathcal{F}_{n}\right]=\lambda h\left(Y_{n}\right) .
$$

For $\lambda>0$, the Martin boundary theory for non-negative $\lambda$-harmonic functions with respect to $Q$ works in the same way as for irreducible Markov chains, since every $x \in T$ can be reached from $o$ with positive probability. Indeed, in analogy with Theorem 3.2, it works for all $\lambda \neq 0$. 
In our situation, the random walk starting at $x \in T$ can visit a vertex $y$ at most once, namely at time $n=d(x, y)$ and when $x$ lies on $\pi(o, y)$. Therefore,

$$
G(x, y \mid \lambda)=F(x, y \mid \lambda) / \lambda=q^{(n)}(x, y) / \lambda^{n+1}, \quad \text { where } n=d(x, y) .
$$

which is non-zero if and only if and only if $y \in T_{x}$. We can define the $\lambda$-Martin kernel as in Eqs. 2.4, and 6.1 yields

$$
K(x, \xi \mid \lambda)=K_{Q}(x, \xi \mid \lambda)= \begin{cases}\frac{\lambda^{|x|}}{q^{(|x|)}(o, x)}, & \text { if } x \in \pi(o, \xi), \\ 0, & \text { otherwise }\end{cases}
$$

It is straightforward that the locally constant function $x \mapsto K_{Q}(x, \xi \mid \lambda)$ is $\lambda$-harmonic. In order to make a notational difference from the "bidirectional" case, we write $\sigma$ instead of $v$ for a finitely additive complex distribution on $\partial T$ as in Eq. 3.2. Then

$$
h(x)=\int_{\partial T} K_{Q}(x, \xi \mid \lambda) d \sigma(\xi)=\frac{\lambda^{|x|}}{q^{(|x|)}(o, x)} \sigma\left(\partial T_{x}\right) .
$$

is $\lambda$-harmonic for $Q$. Conversely, if $h$ is $\lambda$-harmonic, the $\sigma$ can be recovered from $h$ by Eq. 6.4 and a distribution in our sense. We get the following.

Lemma 6.1 For $\lambda \neq 0$, Eq. 6.4 provides a one-to-one correspondence between $\lambda$-harmonic functions for $Q$ and complex distributions on $\partial T$.

As in Section 5, $\lambda$-polyharmonic functions of order $n$ for $Q$ are those which are annihilated by $(\lambda I-Q)^{n}$. We can proceed exactly as in Section 5; the analogue of Proposition 5.1 remains valid, and we have for the $r^{\text {th }}$ derivative with respect to $\lambda$

$$
K_{Q}^{(r)}(x, \xi \mid \lambda)=|x|(|x|-1) \cdots(|x|-r+1) K_{Q}(x, \xi \mid \lambda)
$$

Now the following is obtained in exactly the same way as Theorem 5.3.

Proposition 6.2 For $\lambda \in \mathbb{C} \backslash\{0\}$, every $\lambda$-polyharmonic function $f$ of order $n$ for $Q$ has an integral representation

$$
f(x)=\sum_{r=0}^{n-1} \int_{\partial T} K_{Q}^{(r)}(x, \xi \mid \lambda) d \sigma_{r}(\xi),
$$

where the collection of distributions $\left(\sigma_{0}, \ldots, \sigma_{n-1}\right)$ in the sense of Eq. 3.2 is uniquely determined by $f$. Conversely, every function which has an integral representation as above is $\lambda$-polyharmonic of order $n$ for $Q$.

For real $t$, expand the polynomial

$$
t(t-1) \cdots(t-r+1)=\sum_{k=1}^{r} a_{k, r} t^{k}
$$

By Eq. 6.5, we can re-order the integral terms of Proposition 6.2:

$$
\sum_{r=0}^{n-1} K_{Q}^{(r)}(x, \xi \mid \lambda) d \sigma_{r}(\xi)=K_{Q}(x, \xi \mid \lambda) d \sigma_{0}(\xi)+\sum_{k=1}^{n-1} \sum_{r=k}^{n-1}|x|^{k} a_{k, r} K_{Q}(x, \xi \mid \lambda) d \sigma_{r}(\xi) .
$$


Setting $\bar{\sigma}_{0}=\sigma_{0}$ and $\bar{\sigma}_{k}=\sum_{r=k}^{n-1} a_{k, r} \sigma_{r}$, we get the modified integral representation

$$
f(x)=\sum_{k=0}^{n-1}|x|^{k} \int_{\partial T} K(x, \xi \mid \lambda) d \bar{\sigma}_{k}(\xi),
$$

and the distributions $\bar{\sigma}_{0}, \ldots, \bar{\sigma}_{n-1}$ are uniquely determined by $f$, because the upper diagonal matrix $\left(a_{k, r}\right)_{1 \leq j \leq r \leq n}$ is invertible.

Corollary 6.3 For the forward transition operator $Q$ on $T$ and $\lambda \in \mathbb{C} \backslash\{0\}$ let $f$ be a $\lambda$-polyharmonic function of order $n$. Then $f$ has a representation

$$
f(x)=\sum_{k=0}^{n-1}|x|^{k} h_{k}(x)
$$

where the collection of $\lambda$-harmonic functions $\left(h_{0}, \ldots, h_{n-1}\right)$ for the forward operator $Q$ is uniquely determined by $f$. Conversely, every function of this form is $\lambda$-polyharmonic of order $n$.

This is our analogue of Corollary 5.4 for arbitrary "forward only" transition operators on trees. It generalises [5, Thm. 5.1] to all forward transition operators on countable trees and and all eigenvalues different from 0.

Acknowledgements The authors are grateful to Thomas Hirschler for pointing out that a sign had been mistaken in the derivation formulas of Section 5.

Funding Information Open access funding provided by Graz University of Technology.

Open Access This article is distributed under the terms of the Creative Commons Attribution 4.0 International License (http://creativecommons.org/licenses/by/4.0/), which permits unrestricted use, distribution, and reproduction in any medium, provided you give appropriate credit to the original author(s) and the source, provide a link to the Creative Commons license, and indicate if changes were made.

\section{References}

1. Almansi, E.: Sull'integrazione dell'equazione differenziale $\Delta^{2 n}=0$. Annali di Matematica Serie III 2 , 1-59 (1899)

2. Aronszajn, N., Creese, T.M., Lipkin, L.J.: Polyharmonic Functions. Oxford Math. Monographs. Oxford University Press, New York (1983)

3. Cartwright, D.I., Soardi, P.M., Woess, W.: Martin and end compactifications of non locally finite graphs. Trans. Am. Math. Soc. 338, 679-693 (1993)

4. Cartier, P.: Fonctions harmoniques sur un arbre. Symposia Math. 9, 203-270 (1972)

5. Cohen, J.M., Colonna, F., Gowrisankaran, K., Singman, D.: Polyharmonic functions on trees. Am. J. Math. 124, 999-1043 (2002)

6. Cohen, J.M., Colonna, F., Singman, D.: Distributions and measures on the boundary of a tree. J. Math. Anal. Appl. 293, 89-107 (2004)

7. Dynkin, E.B.: Boundary theory of Markov processes (the discrete case). Russian Math. Surveys 24, 1-42 (1969)

8. Figà-Talamanca, A., Steger, T.: Harmonic analysis for anisotropic random walks on homogeneous trees. Mem. Am. Math. Soc. 110(531) (1994)

9. Guivarc'h, Y.: Sur la loi des grands nombres et le rayon spectral d'une marche aléatoire. Astŕisque 74, 47-98 (1980)

10. Hirschler, T., Woess, W.: Polyharmonic functions for finite graphs and Markov chains. In preparation, TU Graz 
11. Kaimanovich, V.A., Woess, W.: Boundary and entropy of space homogeneous Markov chains. Ann. Probab. 30, 323-363 (2002)

12. Kato, T.: Perturbation Theory for Linear Operators. Grundlehren math Wiss, vol. 132. Springer, Berlin (1966)

13. Picardello, M.A., Woess, W.: Finite truncations of random walks on trees. Symposia Math. 29, 255-265 (1987)

14. Serre, J.-P.: Trees. Springer, Berlin (1980)

15. Woess, W.: Denumerable Markov Chains. Generating functions, Boundary Theory, Random Walks on Trees. European Math. Soc Publishing House (2009)

16. Yosida, K.: Functional Analysis. Springer, Berlin (1980) 